Огляди

DOI: 10.31793/1680-1466.2019.24-4.360

\section{Радиойодтерапия в лечении диффузного токсического зоба}

И.П. Волынец

ГУ «Институт эндокринологии и обмена веществ имени В.П. Комиссаренко НАМН Украины»

\begin{abstract}
Резюме. Диффузный токсический зоб (ДТЗ), или болезнь Грейвса является частой причиной тиреотоксикоза. Варианты лечения болезни Грейвса включают терапию антитиреоидными препаратами (АТП), хирургическое лечение или лечение радиоактивным йодом ('131). Во всем мире ${ }^{131}$ I получил большую популярность в качестве радикального средства лечения тиреотоксикоза. Целью радиойодтерапии является ликвидация тиреотоксикоза путем разрушения гиперфункционирующей ткани щитовидной железы (Щж) и достижение стойкого гипотиреоза. Однако присутствующие радиофобические представления о таком методе лечения тиреотоксикоза создают ряд вопросов на практическом уровне. Этот обзор фокусируется на подходах к терапевтическому применению ${ }^{131}$, в нем обсуждается выбор дозы, подготовка пациента, а также возникновение возможных неблагоприятных состояний, в том числе гипотиреоза, и возможное ухудшение тиреоид-ассоциированной офтальмопатии. Хотя имеются противоречия в лечении тиреотоксикоза ${ }^{131}$, терапия радиоактивным йодом остается безопасным и эффективным методом в лечении болезни Грейвса, достигая высоких показателей эффективности.
\end{abstract}

Ключевые слова: щитовидная железа, болезнь Грейвса, радиоактивный йод, эндокринная офтальмопатия.

Радиоактивный йод $\left({ }^{131} \mathrm{I}\right)$ широко используется для лечения как доброкачественных, так и злокачественных образований щитовидной железы (ЩЖ). Впервые этот метод описан и внедрен в практику в 1940-х годах [1]. Целью терапии ДТЗ является лечение гипертиреоза путем разрушения гиперактивной ткани ЩЖ, достаточное для достижения эутиреоидного или гипотиреоидного состояния [2].

* Адреса для листування (Correspondence): ДУ «Інститут ендокринології та обміну речовин ім. В.П. Комісаренка НАМН України», вул. Вишгородська, 69, м. Київ, 04114, Україна. E-mail: zdovado@ukr.net

о И.П. Волынеи
Имея короткий период полураспада (8,02 суток) и являясь $\beta$-излучающим радионуклидом с максимальной энергией 180 МэВ и пробегом в ткани до 0,8 см, ${ }^{131}$ I остается радионуклидом выбора. Механизм действия радиоактивных изотопов йода является физиологическим. Йод является предшественником тироксина. Радиоактивная форма йода захватывается йодистым транспортером ЩЖ подобно органическому йоду и аналогичным образом обрабатывается. $\beta$-частица разрушает тиреоцит, постепенно приводит к снижению объема ти- 
реоидной ткани, что позволяет контролировать тиреотоксикоз.

Основные показания к радиойодтерапии (РЙТ) включают в себя гипертиреоз как следствие болезни Грейвса, токсического многоузлового зоба и токсической аденомы, а также рак ЩЖ. Противопоказаниями являются беременность, грудное вскармливание и тяжелый неконтролируемый тиреотоксикоз.

Болезнь Грейвса - органоспецифическое аутоиммунное заболевание, которое характеризуется стойкой избыточной продукцией тиреоидных гормонов, гиперплазией и гипертрофией ЩЖ с развитием синдрома тиреотоксикоза, часто сочетаемого с экстратиреоидными нарушениями [3, 4]. Причиной таких нарушений является выработка главным образом антител против рецепторов ТТГ (АТрТТГ), которые при связывании с ними действуют как «аналоги» ТТГ и вызывают стимуляцию рецептора-мишени [4, 8]. Существующие стратегии терапии тиреотоксикоза направлены на нормализацию концентрации тиреоидных гормонов в крови и устранение офтальмологических осложнений, связанных с этой патологией. Это достигается путем применения АТП, угнетающих синтез или высвобождение тиреоидных гормонов, либо путем уменьшения количества функционирующей ткани Щ3.

Основные подходы к терапии радиоактивным йодом заключаются в следующем.

Показания к проведению РЙТ как радикального метода лечения ДТЗ:

- отсутствие стойкой ремиссии на фоне приема тиреостатических препаратов;

- рецидив тиреотоксикоза после правильно проведенного консервативного лечения (непрерывная терапия тиреостатическими препаратами с подтвержденным эутиреозом в течение 12-18 месяцев);

- тяжелые формы ДТЗ с выраженной недостаточностью кровообращения, токсическим гепатитом, психозом;

- наличие сопутствующих заболеваний (врожденные и приобретенные декомпенсированные пороки сердца, инфаркт миокарда, недавно перенесенный инсульт и т.д.), когда проведение оперативного лечения невозможно, а консервативное лечение неэффективно;
- невозможность приема тиреостатических препаратов (лейкопения, аллергические реакции);

- отсутствие условий для консервативного лечения и наблюдения пациента;

- рецидив ДТЗ после предшествующего оперативного лечения при отсутствии эффекта от правильно проведенной медикаментозной антитиреоидной терапии;

- категорический отказ пациента от операции при отсутствии эффекта от проведенной антитиреоидной терапии.

Радиоактивный йод наиболее широко используется для лечения пациентов с болезнью Грейвса в США [2], но поход к дозированию остается спорным. Это связано с различными целями лечения (контроль гипертиреоза или избежание гипотиреоза). По собственным наблюдениям, контроль гипертиреоза при лечении болезни Грейвса радиойодом в 95\% случаев сопровождается развитием гипотиреоза (т.е. гипотиреоз расценивается не как осложнение, а как ожидаемый результат проведенного лучевого лечения). Если рассматривать состояние функциональной автономии ЩЖ как неиммуногенный вариант гипертиреоза, в основе которого лежит феномен автономного захвата йода и продукции гормонов тиреоцитами независимо от влияния ТТГ, то понятно, что радикальное лечение гипертиреоза ${ }^{131} \mathrm{I}$ позволяет выполнять селективную «лучевую резекцию» автономной ткани при минимальном повреждении здоровой паренхимы, приводя к эутиреоидному состоянию пациента в постлучевой период [6].

Для определения адекватных доз ионизирующего излучения в тканях ЩЖ существует два подхода - введение так называемой стандартной (фиксированной) активности, которая гарантирует выключение функции органа-мишени [29, 31], и индивидуальный расчет терапевтической активности, основанный на оценке поглощения дозы радиоактивного изотопа путем определения йод-захвата, эффективного периода его полувыведения, определении объема ЩЖ путем УЗИ.

Согласно рекомендациям Американской тиреоидной ассоциации (АТА), подтвержденным результатами лечения, для пациентов с болезнью Грейвса достаточно для РЙТ активности в 10-15 мКи (370-555 МБк) [7, 10, 15, 
Огляди

29]. Многие же врачи предпочитают использование более высоких активностей ${ }^{131}$ I (20 мКи и более, по некоторым источникам - до 40 мКи), которые приводят к полному разрушению тиреоидной ткани и достижению стойкого гипотиреоза [2]. При дозе в 20 мКи полное разрушение тиреоцитов достигается практически у всех пациентов, и в 75-90\% случаев развивается гипотиреоз [11, 28]. Клиническое значение индивидуального планирования РЙТ остается предметом дискуссий, поскольку полностью стандартизировать способы расчета необходимых доз радиоактивного йода так и не удалось - очень велики индивидуальные различия в чувствительности тканей железы, что частично может быть связано с разным количеством стимулирующих тиреоциты аутоантител против рТТГ в крови [13, 14]. При этом принцип ALARA (as low as reasonably achievable), возможно, является наиболее важным в лучевой терапии, но труднодостижимым, когда идет речь о балансировании быстрого снятия гипертиреоза и отсрочке гипотиреоза. Поэтому многие отказались от вычисляемых доз в пользу фиксированных - 185 МБк, 370 МБк или 555 МБк на основании подтвержденных клинических параметров, таких как размеры ЩЖ [19, 31]. Кроме того, фиксированный режим дозирования является более удобным в использовании.

Адекватная подготовка пациента к РЙТ обеспечивает эффективность процедуры и уменьшает возможные осложнения. Перед проведением лечения следует обсудить с пациентом важные вопросы, которые касаются процедуры лечения, беременности, сроков прекращения медикаментозной антитиреоидной терапии, ее возобновления и возможных осложнений [17]. Перед проведением РЙТ для получения информированного согласия пациента ему необходимо предоставить письменную информацию о вопросах предварительной подготовки, пути введения ${ }^{131} \mathrm{I}$ и формах выпуска (жидкость или капсулы), возможных осложнениях и побочных эффектах, альтернативных вариантах лечения (АТП и оперативное лечение), ожидаемых результатах и цели терапии, риске развития гипотиреоза и пожизненной заместительной терапии L-тироксином. C женщинами необходимо обсудить вопросы, связанные с за- держкой беременности в течение 4-6 месяцев после последней дозы ${ }^{131} \mathrm{I}$, с мужчинами - вопросы, связанные с планированием отцовства (ограничения до 3-4 мес.) Также необходимо письменно уведомить пациента о дате прекращения лечения АТП, дате возобновления приема АТП. Необходимо подробно объяснить пациенту вопросы радиационной защиты - необходимость соблюдения инструкции, мер предосторожности, чтобы избегать ненужного контакта с семьей и коллегами, детьми и беременными. Женщинам детородного возраста обязательно выполнение теста на беременность за 72 часа до начала лечения

Некоторые лекарственные препараты и другие вещества, например рентгенконтрастные, могут препятствовать поглощению йода. Перечень некоторых из таких препаратов приведен в таблице [8, 17].

Пациентам необходимо прекратить использовать йод-содержащие лекарственные препараты и ограничить потребление пищевых продуктов, обогащенных йодом, для обеспечения адекватного захвата радиоактивного йода. Сроки диетических ограничений для доброкачественной патологии ЩЖ окончательно не согласованы, а для пациентов с раком ЩЖ они могут достигать 14-21 дня [4, 8].

Для первичной медикаментозной терапии тиреотоксикоза вследствие болезни Грейвса или как средство для подготовки пациента к окончательной терапии с помощью операции или терапии радиоактивным йодом исполь-

таблица. Перечень лекарств и других веществ, которые могут препятствовать поглощению ${ }^{131}$ |

\begin{tabular}{ll}
\hline Тип лекарства или субстанции & $\begin{array}{l}\text { Продолжительность } \\
\text { прекращения } \\
\text { лечения до РЙт }\end{array}$ \\
\hline Антитиреоидные препараты: & \\
пропилтиоурацил & $1-2$ недели \\
карбимазол, тиамазол & $3-7$ дней \\
мультивитаминные комплексы & 7 дней \\
Агар, раствор Люголя, калия йодид, & $2-3$ недели \\
повидон-йод, йодированный & В зависимости от \\
глицерин & содержания йода* \\
Рентгенконтрастные вещества & $3-4$ недели (при \\
(водорастворимые) & условии нормальной \\
Антиаритмические препараты & почечной функции) \\
группы амиодарона & \\
\hline
\end{tabular}

Примечание: * - количество йода, которое блокирует поступление радиоактивных изотопов на $98 \%$, составляет $>30 \mathrm{Mr} / \mathrm{cym}$. 
зуют карбимазол, тиамазол и пропилтиоурацил $[3,10,11]$. Пациентам старшего возраста, с тяжелым гипертиреозом и сердечно-сосудистыми осложнениями общепринятым является достижение эутиреоза для снижения риска обострения симптомокомплекса тиреотоксикоза на фоне радиационно индуцированного высвобождения гормонов ЩЖ вскоре после проведенного лучевого лечения. Пациентам с более низким риском развития осложнений допустимо достижение состояния субклинического гипертиреоза при условии «прикрытия» сердечно-сосудистой системы бета-блокаторами [7, 29].

Необходимо отметить, что, по данным литературы, применение препаратов группы карбимазола и пропилтиоурацила может способствовать развитию радиорезистентности тиреоцитов и, как следствие, снижению эффективности РЙТ на фоне приема этих препаратов с целью стабилизации гормонального статуса пациента в преддверии лечения [22]. Для преодоления этой проблемы может понадобиться большая доза ${ }^{131} \mathrm{I}$ или прекращение приема препаратов карбимазола за 3-7 дней до терапии, а пропилтиоурацила - за 2 недели [25, 27]. По данным наших наблюдений, этот эффект не оказывает большого влияния на терапевтическое действие радиоактивного йода при гипертиреозе, а вопрос развития радиорезистентности тиреоцитов остается предметом дискуссий.

Возобновление антитиреоидной терапии после лечения радиоактивным йодом осуществляется не ранее чем через неделю после приема ${ }^{131} \mathrm{I}$, поскольку такие препараты могут существенно ускорить выведение изотопов йода из ткани железы, особенно если не все гиперактивные тиреоцити разрушены [14, 16, 27].

Карбонат лития. Литий активно поступает в ЩЖ против градиента концентрации, вероятно, путем активного транспорта, что приводит к заметному снижению высвобождения предварительно сформированного гормона ЩЖ. В более высоких дозах он также может ингибировать реакции органического связывания. По данным литературы, использование карбоната лития в качестве дополнительной терапии является эффективным и применяется с целью задержки высвобождения ${ }^{131} \mathrm{I}$ из ЩЖ, что потенцирует терапевтический эффект последнего [9]. Однако есть и другие данные, которые показывают, что использование лития в течение 3 недель после РЙТ по сравнению с контрольной группой без применения препаратов лития мало влияет на скорость излечения.

Хотя терапия радиоактивным йодом является безопасной и высокоэффективной, некоторые пациенты могут испытывать временное обострение тиреотоксикоза вследствие развития радиационного тиреоидита [12]. Наиболее тревожным является потенциальное ухудшение состояния, связанное с тиреотоксической офтальмопатией.

Орбитопатия Грейвса (ОГ) - основное экстратиреоидное проявление болезни Грейвса, которое представляет собой аутоиммунное поражение, сопровождающееся патологическими изменениями в мягких тканях орбиты с последовательным вовлечением тканей глаза. ОГ является следствием схожести антигенных детерминант в тканях ЩЖ и глазниц, потому любое прогрессирование аутоиммунного поражения ЩЖ приводит к отягощению повреждения тканей глазниц. Ведение таких пациентов осложнено тем фактом, что существующее лечение не влияет на патогенетические механизмы развития заболевания.

Известно, что уровень аутоантител против рТТГ в крови пациентов после воздействия радиоактивными изотопами йода существенно возрастает. Возможно, это связано с реакцией иммунной системы на вызванные внутренним облучением тканей ЩЖ повреждения. В литературе есть данные о сходных показателях прогрессирования ОГ как после проведенной антитиреоидной терапии $(10 \%)$, так и хирургического лечения (16\%), но значительно большее прогрессирование ОГ отмечено после РЙт (до 33\%), чего не наблюдалось в группе пациентов, получавших преднизолон [5]. Один из предлагаемых регламентов рекомендует начать прием преднизолона в дозе 0,3-0,5 мг/кг массы тела за 1 мес. до РЙТ с постепенным снижением дозы на протяжении последующих 3-4 мес. [30]. Впрочем, следует отметить, что во многих случаях предупредить или ослабить активацию орбитопатии позволяют такие меры, как строгий контроль концентрации тиреоидных гормонов в крови до и после воздействия радиойодом и отказ пациента от ку- 
Огляди

рения. Определено, что курение увеличивает риск прогрессирования ОГ после РЙТ, а также способствует уменьшению лечебного влияния лучевой терапии и глюкокортикоидных препаратов [26]. Лечение гипертиреоза с помощью ${ }^{131} \mathrm{I}$ у больных с тяжелой болезнью Грейвса, осложненной выраженной офтальмопатией, не проводится до тех пор, пока тяжесть поражения глаз не будет снижена с помощью курса глюкокортикоидной терапии.

Множество клинических наблюдений показывают, что чаще всего гипотиреоз после разрушения тканей ЩЖ радиоактивными изотопами йода развивается в течение первых 6 месяцев после лечения, иногда возникает отсроченный гипотиреоз в течение первых 2 лет после лечения. Поэтому важно, чтобы после проведения терапии ${ }^{131} \mathrm{I}$ уровни тиреоидных гормонов контролировались ежемесячно. Уровень ТТГ при этом длительное время может оставаться супрессированным. Заместительная терапия левотироксином натрия назначается при клинико-лабораторном подтверждении гипотиреоза.

\section{Список использованной литературы}

1. Shapiro B. Optimization of radioiodine therapy of thyrotoxicosis: what have we learned after 50 years? J Nucl Med. 1993 Oct;34(10):1638-41.

2. Фірсова ММ, Мечев ДС, Полякова НІ, Кащенко ОВ. Досвід застосування I-131 у лікуванні дифузного токсичного зоба. Український радіологічний журнал. 2011;19(3):334-6. (Firsova MM, Mechev DS, Polyakova NI, Kashchenko OV. The experience of applying I-131 in the treatment of diffuse toxic goiter. Ukrayins'kyy radiolohichnyy zhurnal. 2011;19(3):334-6).

3. Campbell K, Doogue M. Evaluating and managing patients with thyrotoxicosis. Australian family physician. 2012; 41(Suppl 8): 564-72.

4. Боднар ПМ, редактор. Ендокринологія: підручник для студ. вищих мед. навч. закладів. вид. 3-є, переробл. та доповн. Вінниця: Нова Книга; 2013. 480 c. (Bodnar PM, editor. Endocrinology: a textbook for the students of higher med educational institutions. vyd. 3-ye, pererobl. ta dopovn. Vinnytsya: Nova Knyha; 2013. $480 \mathrm{~s}$.).

5. Шеремета МС, Беловалова ИМ, Свириденко НЮ. Радиойодтерапия болезни Грейвса как фактор риска эндокринной офтальмопатии. Проблемы эндокринологии. 2009;55(1):51-5. (Sheremeta MS, Belovalova IM, Sviridenko NYu. Radioiodine therapy for Graves' disease as a risk factor for endocrine ophthalmopathy. Problemy endokrinologii. 2009;55(1):51-5).

6. Дедов ИИ, Мельниченко ГА, редакторы. Эндокринология: национальное руководство. Москва: ГЭОТАР-Медиа, 2008. 1072 c. (Dedov II, Melnichenko GA, editors. Endocrinology: national leadership. Moscow: GEOTAR-Media; 2008. 1072 p.).

7. Фадеев ВВ. Диагностика и лечение болезни Грейвса. Медицинский совет. 2014;4:44-9. (Fadeev VV. Diagnosis and treatment of Graves' disease. Meditsinskiy sovet. 2014;4: 44-9).

8. Дедов ИИ, Мельниченко ГА, редакторы. Заболевания щитовидной железы. Генри М Кроненберг, Шломо Мелмед, Кеннет С Полонски, П Рид Ларсен; пер. с англ. Москва: ООО «Рид Элсивер»; 2010. 392 с. (Серия «Эндокринология по Вильямсу»).
Перевод изд. Williams Textbook of Endocrinology, 11th edition. (Dedov II, Melnichenko GA, editors. Diseases of the thyroid gland. Henry M Kronenberg, Shlomo Melmed, Kenneth S Polonsky, P Reed Larsen; transl. from English. Moscow: Reed Elsiver LLC; 2010. 392 p.) (Series «Endocrinology by Williams»). Translation ed. Williams Textbook of Endocrinology, 11th edition).

9. Древаль АВ, Цыб АФ, Нечаева ОА, Комердус ИВ, Дроздовский БЯ. Влияние адъювантной терапии карбонатом лития на результаты лечения радиоактивным йодом больных диффузным токсическим зобом. Проблемы эндокринологии. 2007; 53(6):15-9. (Dreval' AV, Tsyb AF, Nechayeva OA, Komerdus IV, Drozdovskiy BYa. The effect of adjuvant lithium carbonate therapy on the results of treatment with radioactive iodine in patients with diffuse toxic goiter. Problemy endokrinologii. 2007; 53(6):15-9).

10. Черенько СМ. Сучасні підходи до діагностики й лікування різних форм тиреотоксикозу: огляд останніх рекомендацій Американської тиреоїдної асоціації й Американської асоціації клінічних ендокринологів. Медична газета «Здоров'я України». 2012: 64. (Cheren'ko SM. Modern approaches to the diagnosis and treatment of various forms of thyrotoxicosis: an overview of the latest recommendations of the American Thyroid Association and the American Association of Clinical Endocrinologists. Medychna hazeta «Zdorov'ya Ukrayiny». 2012:64).

11. Караченцев ЮИ, Казаков АВ, Кравчун НА, Ильина ИМ. 100 избранных лекций по эндокринологии. [2-й выпуск]. Харьков: «C.A.M.»; 2014. 1000 c. (Karachentsev YuI, Kazakov AV, Kravchun NA, Il'ina IM, eds. 100 selected lectures on endocrinology [2nd edition]. Khar'kov: «S.A.M.»; 2014. 1000 p.).

12. Gregory A, Brent MD. Graves' disease. N Engl J Med. 2008; 359(13): 1407-12.

13. Liu M, Jing D, Hu J, Yin S. Predictive factors of outcomes in personalized radioactive iodine (131I) treatment for Graves' disease. Am J Med Sci. 2014; 348(4): 288-93.

14. Iagaru A, McDougall IR. Treatment of thyrotoxicosis. J Nucl Med. 2007; 48(3):379-89.

15. Montesano T, Toteda M, D'Apollo R, Di Nicola AD, Acqualagna G, Ciancamerla M. 131 I therapy and Graves' disease in a long term observation: euthyroidism is a suitable goal. Our experience. Clin Ter. 2014;165(2):139-44.

16. Read CH, Tansey MJ, Menda Y. A 36-years retrospective analysis of the efficacy and safety of radioactive iodine in treating young Graves patients. J Clin Endocrinology. 2004;89(9):4229-33.

17. Mumtaz M, Lin LS, Hui KC, Mohd Khir AS. Radioiodine I-131 for the therapy of Graves' Disease. Malays J Med Sci. 2009 Jan-Mar; 16(1):25-33.

18. Walter MA, Christ-Crain M, Schindler C, Müller-Brand J, Müller B. Outcome of radioiodine therapy without, on or 3 days off carbimazole: a prospective interventional three-group comparison. Eur J Nucl Med Mol Imaging. 2006;33(6):730-7.

19. Willegaignon J, Sapienza MT, Coura-Filho GB, Watanabe T, Traino AC, Buchpiguel CA. Graves' disease radioiodine therapy: choosing target absorbed doses for therapy planning. Med Phys. 2014 Jan; 41(1):012503.

20. Alexander EK, Larsen PR. High dose 131-I therapy for the treatment of hyperthyroidism caused by Graves' disease. J Clin Endocrinol Metab. 2002; 87:1073-7.

21. Yoshihara A, Noh JY, Watanabe N, Iwaku K, Kobayashi S, Suzuki M, et al. Lower incidence of postpartum thyrotoxicosis in women with Graves' disease treated by radioiodine therapy than by subtotal thyroidectomy or with antithyroid drugs. Clin Nucl Med. 2014;39(4):326-9.

22. Bonnema SJ, Bennedbaek FN, Veje A, Marving J, Hegedüs L. Propylthiouracil before 131I therapy of hyperthyroid diseases: effects on cure rate evaluated by randomised control trial. J Clin Endocrinol Metab. 2004 Sep; 89(9):4439-44.

23. Kalinyak JE, McDougall IR. How should the dose of iodine-131 be determined in the treatment of Graves' hyperthyroidism? J Clin Endocrinol Metab. 2003; 88(3):975-7.

24. Leslie WD, Ward L, Salamon EA, Ludwig S, Rowe RC, Cowden EA. A randomized comparison of radioiodine doses in Graves' hyperthyroidism. J Clin Endocrinol Metab. 2003; 88(3):978-83.

25. Burch HB, Solomon BL, Cooper DS, Ferguson P, Walpert N, Howard R. The effect of antithyroid drug pretreatment on acute 
changes in thyroid hormone levels after (131)I ablation for Graves' disease. J Clin Endocrinol Metab. 2001;86(7):3016-21.

26. Bartalena L, Bogazzi F, Tanda ML, Manetti L, Dell'Unto E, Martino E. Cigarette smoking and the thyroid. Eur J Endocrinol. 1995 Nov;133(5):507-12.

27. Walter MA, Briel M, Christ-Crain M, Bonnema SJ, Connell J, Cooper DS, et al. Effects of antithyroid drugs on radioiodine treatment: systematic review and metaanalysis of randomized controlled trials. Br Med J. 2007; 334 (7592):514-7.

28. Razvi S, Basu A, McInture EA, Wahid ST, Bartholomew PH, Weaver JU; Queen Elizabeth Hospital, Gateshead, UK. Low failure rate of fixed administered activity of $400 \mathrm{MBq} 131$-I with pre-treatment with carbimazole for thyrotoxicosis: the Gateshead Protocol. Nucl Med Commun. 2004 Jul;25(7):675-82.

29. Ross DS, Burch HB, Cooper DS, Greenlee MC, Laurberg P, Maia AL, et al. 2016 American Thyroid Association Guidelines for Diagnosis and Management of Hyperthyroidism and other causes of thyrotoxicosis. Thyroid. 2016 Oct;26(10):1343-421.

30. Bartalena L, Baldeschi L, Boboridis K, Eckstein A, Kahaly GJ, Marcocci C, et al. The 2016 European Thyroid Association/ European Group on Graves' Orbitopathy Guidelines for the Management of Graves' Orbitopathy. Eur Thyroid J. 2016 Mar;5(1):9-26

31. Kahaly GJ, Bartalena L, Hegedüs L, Leenhardt L, Poppe K, Pearce SH. 2018 European Thyroid Association Guideline for the Management of Graves' Hyperthyroidism. Eur Thyroid J. 2018;7(4):167-86.

(Надійшла до редакції 08.11.2019р.)

\section{Радіойодтерапія в лікуванні дифузного токсичного зоба}

\section{І.П. Волинець}

ДУ «Інститут ендокринології та обміну речовин імені В.П. Комісаренка НАМН України»

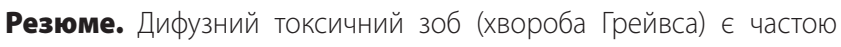
причиною розвитку симптомокомплексу тиреотоксикозу. Варіанти лікування хвороби Грейвса включають лікування антитиреоїдними препаратами, хірургічне та лікування радіоактивним йодом. В усьо- му світі ${ }^{131}$ отримав велику популярність для радикального лікування тиреотоксикозу, унаслідок якого руйнується гіперфункціонуюча тканина щитоподібної залози та досягається евтиреоз або стійкий гіпотиреоз. Цей огляд сфокусовано на підходах до терапевтичного використання ${ }^{131}$ І, у ньому обговорюються вибір дози, особливості підготовки пацієнта, а також можливі несприятливі стані, у т.ч. гіпотиреоз і погіршення перебігу тиреоїд-асоційованної офтальмопатії.

Ключові слова: щитоподібна залоза, хвороба Грейвса, радіоактивний йод, тиреотоксична офтальмопатія.

\section{Radioactive iodine treatment for diffuse toxic goider}

\section{I.P. Volynets}

State Institution «V.P. Komisarenko Institute of Endocrinology and Metabolism of National Academy of Medical Sciences of Ukraine»

Abstract. Graves' disease is the most common cause of thyrotoxicosis. There are three distinct treatments for these conditions, antithyroid medications, thyroidectomy and radioiodine. lodine-131 therapy is the method of choice for treating new-onset of Graves' disease in many countries. The purpose of radioiodine therapy is to destroy the thyroid tissue with increased function, resulting in euthyroidism or hypothyroidis. The different approaches to application of radioactive iodine treatment are described. This review focuses on treatment approaches for dose selection in radioiodine therapy, patient preparation and the development of possible side effects, including one of the more controversial progression aspects of thyroid associated ophthalmopathy. Treatment with ${ }^{131}$ I has been found to be cost-effective, safe and reliable.

Keywords: thyroid gland, diffuse toxic goiter (Graves' disease), radioactive iodine, Graves' ophthalmopathy. 


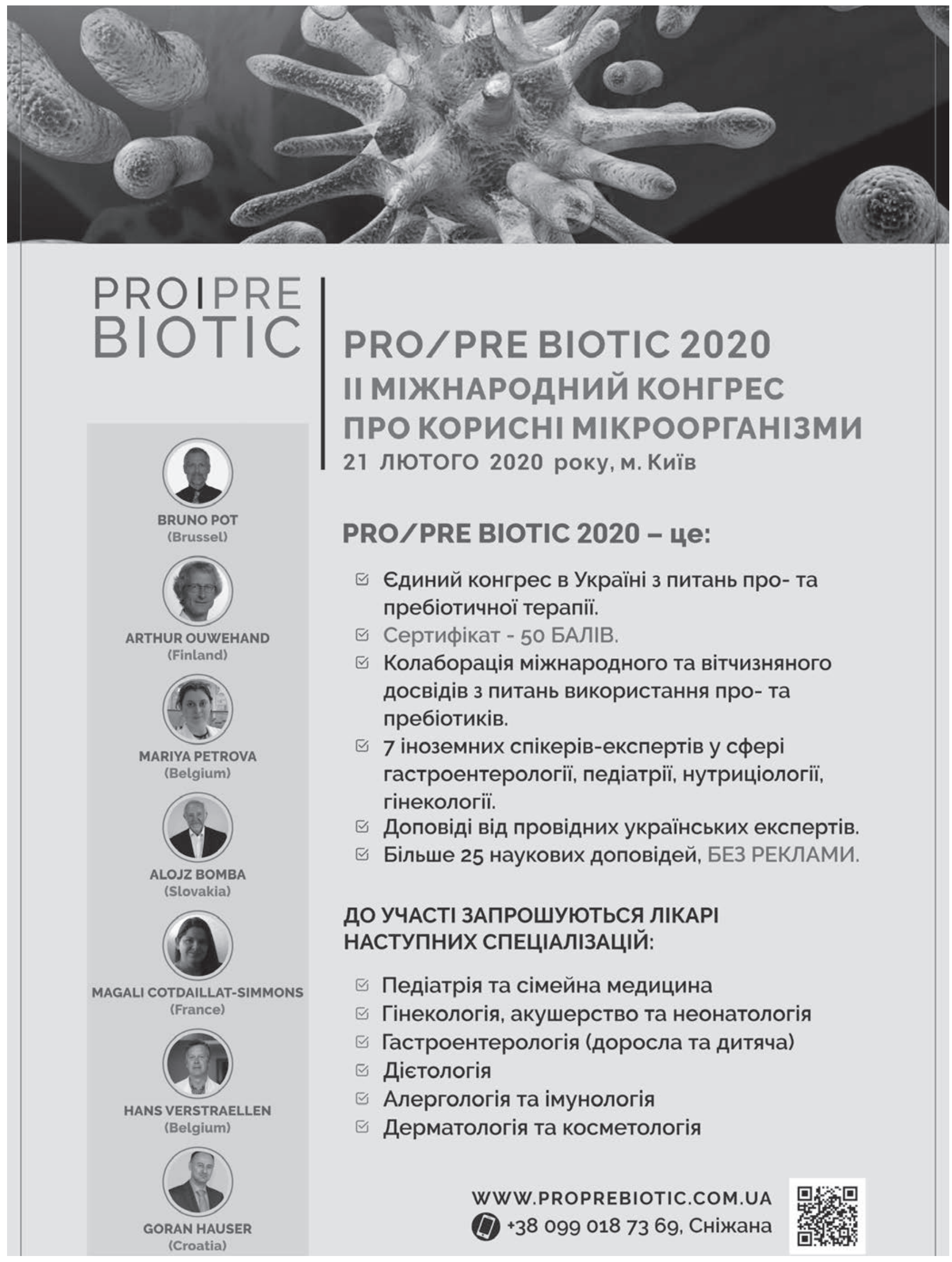

